\title{
TEORIA DAS RELAÇÕES INTERPESSOAIS EM ENFERMAGEM DE PEPLAU: ANÁLISE E EVOLUÇÃO
}

\author{
Silvana Sidney Costa Santos ${ }^{15}$ \\ Maria Miriam Lima da Nóbrega ${ }^{16}$
}

RESUMO: Este é um estudo descritivo analítico onde procura-se analisar a Teoria das Relações Interpessoais em Enfermagem, de Hildegard E. Peplau. seguindo o modelo conceitual de análise e evolução proposto por Thibodeau. Descreve os conceitos de pessoa, ambiente, saude e enfermagem, a origem e a metodologia utilizada para a formação dessa teoria, além da sua generalização, utilidade, aceitação e significado dentro da enfermagem. Destaca a importância para a sua aplicação na prática dos enfermeiros em vista de ser o processo interpessoal uma etapa primordial na assistência de enfermagem.

UNITERMOS: Teoria de enfermagem - Relação interpessoal - Enfermagem.

\section{INTRODUÇÃO}

$\mathrm{Na}$ década de 60, com a evolução da abordagem científica na enfermagem, os enfermeiros começaram a questionar os propósitos da profissão e o valor da prática de enfermagem tradicional e de natureza intuitiva. Segundo Fawcett (1987), estes questionamentos levaram os enfermeiros a discutirem e a escreverem sobre suas bases filosóficas para a prática, ou seja, ao rem desenvolvimento das teorias de enfermagem e conseqüentemente a identificação de elementos comuns que inclui a natureza da enfermagem, o individuo como recipiente do cuidado, a sociedade e ambiente e a saúde. Contudo, merece ser ressaltado que, apesar do consenso entre estes elementos, as teorias de enfermagem diferem em seus caminhos $e$ especificidade na qual define seus conceitos. Para a utilização das teorias de enfermagem, como norteadora da prática de enfermagem, é imprescindivel que o enfermeiro conheça o seu desenvolvimento e introjete os conceitos de acordo com as especificidades de cada teoria.

15 Prolessora Adjunto do Departamento de Enfermagem de Saúde Pública c Psiquiatria da Universidade Federal da Paraíba. Docente do Mestrado de Enfermagem em Saúde Pública UFPB.

16 Professora Adjunto do Departamento de Enfermagem de Saúde Pública c Psiquiatria da Universidade Federal da Paraíba. Docente do Mestrado de Enfermagem em Saúde Pública UFPB. 
Para facilitar esta tarefa, vários enfermeiros têm desenvolvido estudos para análise, avaliação e crítica das teorias, objetivando a compreensão dos conceitos e ao mesmo tempo possibilitando a sua utilização na prática. no ensıno e na pesquisa. Entre as várias propostas de modelos de análise das teorias destacam-se os trabalhos de Stevens, Meleis, Chinn, Thibodeau, e Walker e Avant (Cianciarullo, 1987)

Dentre as teorias de enfermagem existentes, a Teoria das Relações Interpessoais em Enfermagem foi publicada em 1952 no livro Interpersonal Relations in Nursing, da $\mathrm{Dr}^{a}{ }^{a}$ Hildegard Elizabeth Peplau, o qual, segundo Taylor (1990), revolucionou, de muitas formas, o ensino e a pratica da enfermagem psiquiátrica nos Estados Unidos. A partir de uma leitura preliminar podemos constatar que nesta teoria Peplau enfoca o potencial terapêutico do relacionamento de pessoa-para-pessoa e mostra que, embora o enfermeiro possa administrar medicamentos e auxiliar em outros tratamentos psiquiátricos, o principal modo como ele influencia diretamente no atendimento ao paciente é através do uso que faz de si mesmo enquanto lida com um cliente em interações individuais. Para lidar em interações individuais, a referida autora apresentou as fases da relação interpessoal, os papéis nas situações de enfermagem e os métodos para o estudo da enfermagem como um processo interpessoal com foco no desenvolvimento das relações entre o paciente e o enfermeiro, os quais têm como base o interacionısmo, a fenomenologia, o existencialismo filosófico e o humanismo.

A partir desta constatação, associado ao fato de sermos enfermeiras psiquiátricas, decidimos desenvolver este estudo objetivando analisar a Teoria das Relações Interpessoais em Enfermagem de Hildegard E. Peplau, a partir do modelo conceitual de análise e evolução de Thibodeau, visando a utilização desta análise como suporte/conceituação teórica no trabalho de dissertação da autora principal do estudo. A escolha por este modelo de avaliação se deu em virtude do mesmo permitir a avaliação por mais de uma pessoa, com diferentes perspectivas e com diferentes fins.

\section{METODOLOGIA}

Trata-se de um estudo descritivo analítico, realizado a partir do levantamento na Rede de computadores da Biblioteca da UFPB, na Biblioteca da FENSG/UPE, na Sala de Leitura do Mestrado de livros e periódicos que contemplassem a Teoria das Relações Interpessoais de Peplau. A partir desta tentativa, foi feito uma leitura seletiva do material coletado de acordo com os objetivos propostos, fichamento em resumo critico-analitico dos textos selecionados para posterior leitura interpretativa dos conteúdos dos diferentes autores pesquisados e considerações dos pesquisadores sobre o fenômeno evidenciado e por fim a análise obedecendo as seguintes etapas do modelo de análise e evolução de Thibodeau (1988):

I - Análise dos conceitos: pessoa, ambiente saúde e enfermagem, enfatizando os objetivos da enfermagem e o processo de enfermagem e a relação entre os quatro conceitos principais. 
II - Identificar a origem do problema.

III - Identificar a metodologia para desenvolver o modelo atentando para a consistência interna e a validação empírica.

IV - Verificar a generalização da teoria.

V - Descrever a utilidade, a aceitação e o significado do modelo teórico para a enfermagem.

\section{TEORIA DE PEPLAU E OS QUATRO CONCEITOS FUNDAMENTAIS}

\section{PESSOA}

Peplau define a pessoa em termos de homem e este homem como um organismo que "luta a seu modo para reduzir a tensão gerada pelas necessidades". A autora utiliza ainda a definição de paciente como sendo o individuo que necessita de cuidados de saúde, considerando o ser humano um organismo vivo em estado de equilíbrio instável que luta para atingir um estado de equilibrio perfeito, o que só atingirá pela morte (George,1993).

\section{AMBIENTE}

Para Tomey (1989), o ambiente está implicitamente definido quando Peplau afirma que "há forças fora do organismo e no contexto da cultura que influem no paciente", mas a teorista não se refere, diretamente, a sociedade/ambiente; ela na verdade estimula o profissional de enfermagem a levar em conta a cultura e os costumes do paciente. Acreditamos que a escassa percepção de Peplau acerca da sociedade/ambiente constitui uma das principais limitações da sua teoria.

\section{SAÜDE}

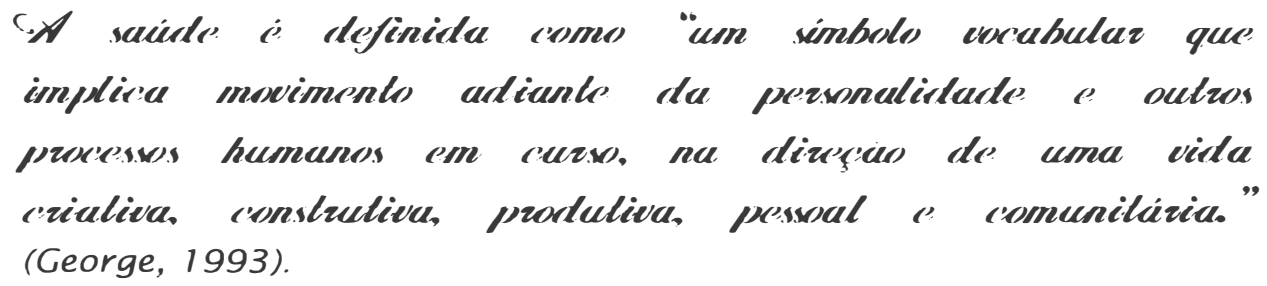

\section{ENFERMAGEM}

Peplau (1988) considera a enfermagem uma arte terapêutica e um processo interpessoal, onde cada indivíduo é visto como um ser bio-psicosócio-espiritual, dotado de crenças, costumes, usos e modos de vida voltados para determinada cultura e ambiente diversificado. Para a autora, a enfermagem é uma relação humana entre um individuo que está doente ou 
necessitado de serviços de saúde e um enfermeiro preparado para reconhecer e para responder às necessidades de ajuda do paciente.

Segundo Stefanelli (1987), Peplau considera como elementos básicos ou variáveis, nas situações de enfermagem, as necessidades humanas básicas, a frustração, o conflito e a ansiedade, que devem ser tratados, no relacionamento enfermeira-paciente de modo a favorecer o crescimento, ou seja, o desenvolvimento saudável da personalidade. Peplau encara a Enfermagem como uma "força de amadurecimento e um instrumento educativo" (GEORGE, 1993), uma vez que à medida que o enfermeiro assiste o paciente utilizando a relação interpessoal como principal ferramenta, cresce, conhecendo-se melhor, e ajuda o paciente a crescer também.

\section{5 - COMPARAÇÃO ENTRE OS QUATRO CONCEITOS}

Comparando a relação entre os quatro conceitos apresentados, verifica-se que todos se adequam à visão do final da década de 40 e do início da década de 50, quando o livro de Peplau foi publicado, ou seja, não havia ainda uma ampla influência ambiental sobre 0 individuo, pois se focalizava mais as tarefas psicológicas individuais do ser necessitado de ajuda, cuja ajuda era encontrada num hospital. Observa-se, então, que os quatro conceitos da Teoria das Relações Interpessoais de Peplau baseiam-se nas teorias desenvolvimentais, dos conceitos de adaptação à vida e das reações aos conflitos das relações interpessoais.

\section{6 - OUTROS CONCEITOS DA TEORIA}

Os outros conceitos da teoria de Peplau incluem crescimento, desenvolvimento, comunicação e papel. Para Boniean (1987), a comunicação é um processo de solução de problema usado pelo enfermeiro na relação com o cliente. Este processo é colaborativo, no qual o enfermeiro pode assumir muitos papeis para ajudar o cliente no atendimento de suas necessidades e desta forma colaborando com o seu crescimento e desenvolvimento.

Para atender a assistência de enfermagem, Peplau desenvolveu uma série de passos que seguem determinado padrão terapêutico e em cujo centro está a relação do enfermeiro com o paciente, a qual é flexivel, está baseada nos principios científicos e aquisição de papéis. Estes passos são: (1) orientação, (2) identificação, (3) exploração e (4) solução, os quais superpõem-se e interrelacionam-se à medida que o processo evolui na direção de uma solução (George, 1993).

A fase de orientação é o início da relação interpessoal, e ocorre quando o paciente/família percebe a necessidade de ajuda. Tanto o enfermeiro quanto o paciente trazem suas bagagens anteriores (cultura, valores, idéias préconcebidas, etc.), que devem ser levadas em consideração; ambos têm papel igualmente importante na interação interpessoal. Existe nesta fase todo o aspecto educativo da relação. 
$\mathrm{Na}$ fase de identificação o paciente reage seletivamente no enfermeiro; ambos precisam esclarecer as percepções e expectativas mútuas. No final desta fase o paciente começa a lidar com o problema, o que diminui a sensação de impotência e desesperança do enfermeiro, criando uma atitude de otimismo.

$\mathrm{Na}$ fase de exploração o paciente começa a sentir-se parte integrante do ambiente provedor de cuidados e pode necessitar "demais" do enfermeiro como uma forma de "conseguir atenção". É importante para o enfermeiro saber usar os princípios para técnicas de entrevista. O paciente poderá apresentar um conflito entre dependência-independência. $O$ enfermeiro precisa oferecer uma atmosfera sem ameaças e utilizar adequadamente a comunicação: esclarecendo, escutando, aceitando e interpretando corretamente; assim o enfermeiro ajuda o paciente na exploração de todos os caminhos da saúde.

A fase de solução é a última do processo interpessoal. Presume-se que as necessidades do paciente já foram satisfeitas através dos esforços cooperativos do enfermeiro e do paciente. Ocorre a dissolução do elo da relação terapêutica.

$\mathrm{Na}$ execução destas fases são assumidos diferentes papéis profissionais, os quais podem ser descritos da seguinte maneira: Professor - aquele que partilha conhecimentos a respeito de uma necessidade ou interesse; Recurso - aquele que oferece informações especificas, necessárias, que auxiliam na compreensão de um problema ou situação, de determinadas habilidades e atitudes, auxilia outra pessoa no reconhecimento, enfrentamento, aceitação e solução de problemas que interferem em sua capacidade de viver feliz e eficientemente; Líder - aquele que executa o processo de início e manutenção das metas do grupo, através da interação; Especialista Técnico - aquele que providencia cuidados físicos, exibindo habilidades clínicas e que apresenta a capacidade de utilizar equipamentos nessa tarefa; e Substituto - aquele que assume o lugar do outro (George, 1993).

Peplau descreve ainda quatro experiências psicobiológicas: necessidades, frustração, conflito e ansiedade. Estas experiências proporcionam energia que é transformada em alguma forma de ação. Peplau não utiliza conceitos teóricos de enfermagem para identificar e explicar estas experiências que compelem respostas destrutivas ou construtivas provenientes do enfermeiro e do paciente. Este entendimento providencia uma base para formação de objetivo e intervenções de enfermagem (Tomey, 1989). 


\section{Comparação do Processo de Enfermagem e as Fases da Teoria de Peplau}

\begin{tabular}{|c|c|}
\hline Processo de Enfermagem & Fases de Peplau \\
\hline 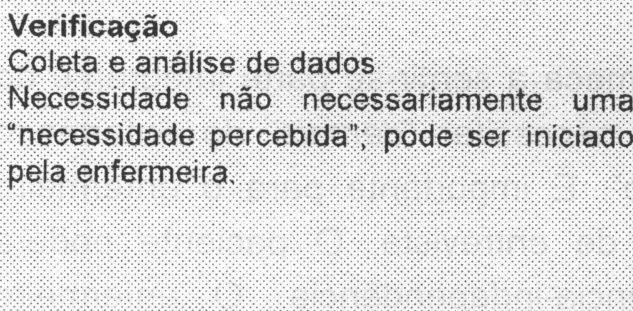 & 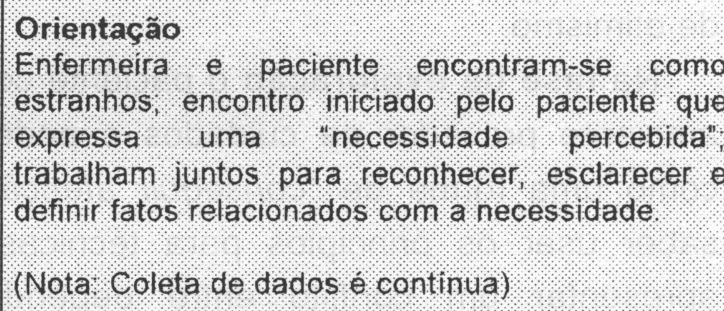 \\
\hline $\begin{array}{l}\text { Diagnóstico de Enfermagem } \\
\text { Metas nutuamente fixadas }\end{array}$ & $\begin{array}{l}\text { Identificacăo } \\
\text { Alxacáo inter dependente de netas. Paciente tem } \\
\text { a sensaçáo de pertencer e reage, seletivamente, } \\
\text { aqueles que podem satisfazer as necessidades. } \\
\text { Iniciada pelo paciente. }\end{array}$ \\
\hline 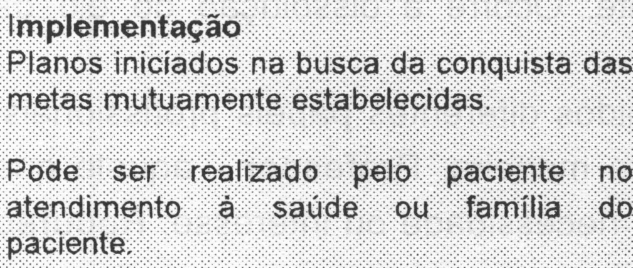 & 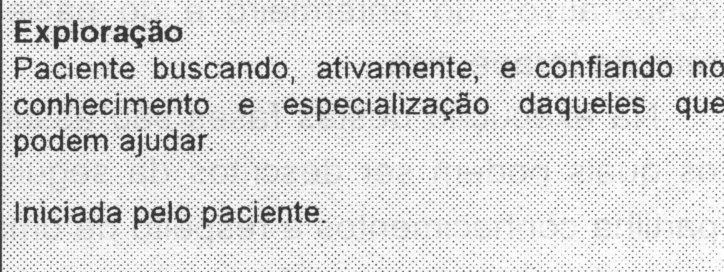 \\
\hline 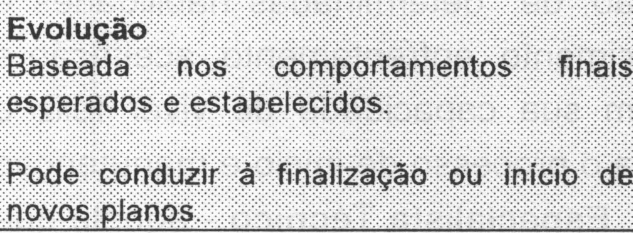 & 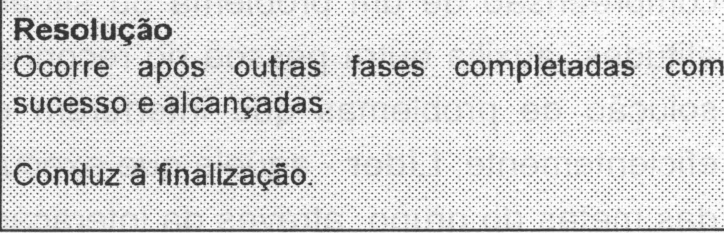 \\
\hline
\end{tabular}

Fonte: GEORGE, 1993

Observamos que há uma significativa relação entre as fases do trabalho teórico de Peplau e o que preconiza o processo de enfermagem, pois há um continuum em ambos que enfatizam as seqüências lógicas da interação terapêutica, onde são incluídas como ferramentas básicas à observação, comunicação e registros utilizados pelo enfermeiro no desenvolver de suas ações (George, 1993).

\section{ORIGEM DA TEORIA INTERPESSOAL DE PEPLAU}


Peplau usou conhecimento emprestado da ciência comportamentalista e do que possa ser chamado de modelo psicológico (Tomey,1989). A composição conceitual dos relacionamentos interpessoais procura desenvolver as habilidades do enfermeiro usando a orientação através dos eventos de significância psicológica, sentimentos e comportamentos do paciente. A teoria interpessoal de Harry Stack Sullivam; a teoria da psicodinâmica de Sigmund Freud; a teoria de Abraham Maslow da motivação humana; o trabalho de Neal Elgar Miller voltado para a teoria da personalidade, mecanismos de ajuste, psicoterapia e princípios do aprendizado social são alguns dos muitos recursos que Peplau usou no desenvolvimento da sua composição conceitual (TOMEY, 1989).

Peplau estava comprometida em incorporar conhecimentos já estabelecidos dentro da composição conceitual dela, para assim desenvolver uma teoria baseada no modelo de enfermagem, o que conseguiu com sua Teoria das Relações Interpessoais.

Cumpre destacar a importância do referencial das teorias interpessoais para a enfermagem ao se colocar como uma tentativa de mudança de um olhar clínico, meramente, para um olhar compreensivo, da enfermeira dirigido ao paciente (AGUIAR, 1995).

\section{METODOLOGIA DA TEORIA DAS RELAÇÕES INTERPESSOAIS}

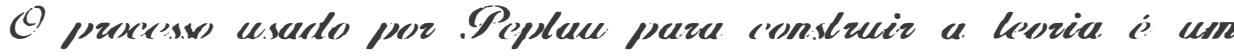

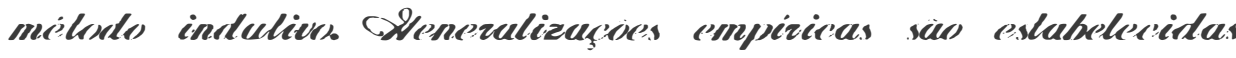

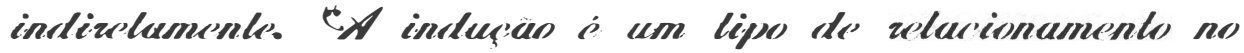
qual a pesso obsewa eventos empivicos "genezalizaveis de

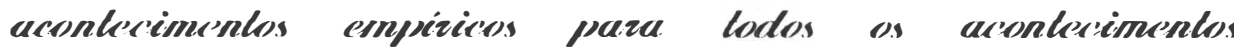
similuze" (Tomey, 1989)

D)e as'ruts cism .Peplau, "siluapoes de enfermagem propsuzisonasn usn easnpos de obsewasoses dos quais apsenas

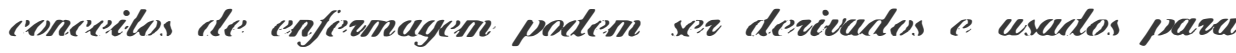
- apserfiegrasnents do lrabulho profisional" (Tomey, 1989).

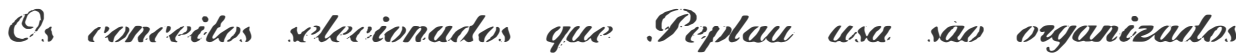

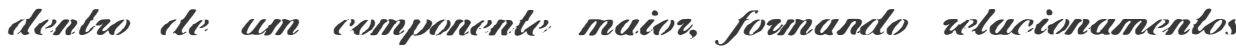

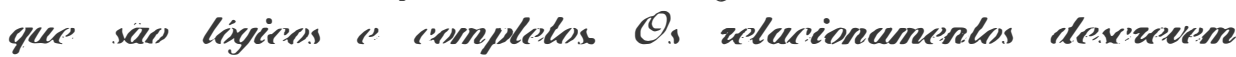

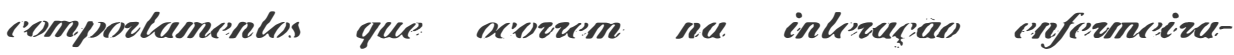
pacienk.

Percebe-se ainda que, além do método indutivo, Peplau utilizou também conhecimentos mais amplos, de outras ciências, daí poder-se afirmar que o 
método que melhor responde pela Teoria das Relações Interpessoais é o método hipotético-dedutivo.

\section{UTILIDADE, ACEITAÇÃO E SIGNIFICADO DA TEORIA DAS RELAÇÕES INTERPESSOAIS}

Peplau trouxe uma nova perspectiva, um novo método, uma fundamentação teoricamente baseada para a prática de enfermagem no trabalho terapêutico com pacientes (Tomey,1989). O trabalho de Peplau é responsável por uma segunda mudança de ordem na cultura da enfermagem, pois "possibilitou a compreensão de que o cuidado envolve contato, relação e interação" (Aguiar. 1995).

As críticas ao modelo indicam a falta de desenvolvimento dos sistemas sociais que poderiam ampliar 0 conhecimento base para se entender 0 problema do paciente. Apesar de Peplau ter usado as teorias interpessoal e intrapessoal de Sullivan e Freud como base teórica para o seu modelo. não levou em consideração os interrelacionamentos entre homem e sociedade (Tomey, 1989).

A teoria de Peplau é muito aceita em enfermagem psiquiátrica, saúde mental e enfermagem clínica, tanto ao nivel de ensino, da pesquisa e com menor intensidade na prática. A teoria de Peplau pode ser considerada precisa, porém há necessidade de ser mais utilizada a fim de que este grau de precisão aumente.

Quanto ao significado, Peplau é uma das primeiras teoristas desde Nightingale a apresentar uma teoria para enfermagem. Por isso o trabalho dela pode ser considerado como pioneiro dando à enfermagem um método significativo da prática do autodirecionamento numa época em que a medicina domina o campo do cuidado à saúde.

\section{CONSIDERAÇÕES FINAIS}

Embora o livro de Peplau tenha sido publicado na década de 50, quatro décadas atrás, constatamos que os conceitos da teoria continuam dando direção para a prática da enfermagem, para a educação e a pesquisa. Este fato se explica por ter sido esta teoria desenvolvida co base na realidade, o que tem favorecido a relação existente entre a teoria e os dados empíricos, os quais permitem a validação e verificação da teoria por outros cientistas.

A Teoria de Peplau lida com relacionamentos do processo interpessoal enfermeiro - paciente e experiências psicobiológicas. Cada um desses relacionamentos é então desenvolvido dentro da teoria, de uma forma compreensivel. Dai a teoria ser descrita como uma junção de alto grau de simplicidade. A teoria de Peplau é adaptável apenas na enfermagem em locais onde possa haver a comunicação entre o paciente e o enfermeiro. $O$ seu uso é limitado no trabalho com pacientes inconscientes, recém-nascidos e alguns pacientes idosos. Portanto, nesta teoria a qualidade da generalidade não é encontrada. Desta forma podemos concluir que o trabalho de Peplau 
tem fornecido uma contribuição significante para a base de conhecimentos da enfermagem.

ABSTRACT: This is a bibliographic study where the authors tried to analyse the interpersonal relationships of nursing from Hildegard E. Peplau following the conceptual pattern of analysis and evolution proposed by Thibodeau. It describes the concepts of person, enviroment, health and nursing; the source and procedures used to this theory formation; and its generalization, use, acceptance and significance for nursing. It shows the importance of its application in nursing practice because the interpersonal process is a primary stage in nursing assistance.

KEYWORD: Theory; Interpersonal Relationship - Nursing.

\section{REFERÊNCIAS BIBLIOGRÁFICAS}

1. AGUIAR, N. G. G. Revisando a literatura como forma de repensar a prática da enfermeira no campo da psiquiatria. apresentado ao $47^{\circ}$ Congresso Brasileiro de Enfermagem, Goiânia, 1995, 18p. (Mimeo).

2. BONIEAN, L. Personality theories and mental health. In: COOK, J. S., FONTAINE, K. L. Essentials of mental health nursing. Califórnia: Addison-Wesley Company, 1987. P.94

3. CIANCIARULlO, T. W. Teoria das Necessidades Humanas Básicas - um marco indelével na enfermagem brasileira. Rev. Esc. Enf. USP. São Paulo, v. 21, n. Especial, p. 100-107, 1987.

4. FAWCETT, J. Analysis and evaluation of conceptual models of nursing. Philadelphia: F.A. Davis, 1987. p.34-54

5. GEORGE, J. B. Teorias de enfermagem, a base para a prática da profissão. Porto Alegre: Artes Médicas, 49-62, 1993. 
6. PEPLAU, H. E. Interpersonal relations in Nursing: a conceptual frame of references for psychodynamic nursing. Kingdon: MacMillan Educacion, 1988, p.3-16.

7. StefANELLI, M. C. Ensino de técnicas de comunicação terapêutica enfermeira-paciente: referencial teórico (Parte II). Rev. Esc. Enfermagem da USP, v. 21, n. 2, p.107-115, ago, 1987.

8. TAYLOR, C. M. Interações enfermeira-cliente. In: Fundamentos de enfermagem psiquiátrica. 13. ed. Porto Alegre: Artes Médicas. p.71, 1990.

9. THIBODEAU, J. A. Nursing Models: analysis and evolution. Connecticut: University of Connecticut, 1988.

10. TOMEY, A M. Nursing Theorists and their Work. 2.ed. Toronto: The C.V. Mosby Company. p.203-211, 1989. 